\title{
A influência da alimentação no envelhecimento e nas desordens estéticas: uma
}

\section{revisão integrativa}

\author{
The influence of food on aging and aesthetic disorders: an integrative review \\ La influencia de la alimentación en el envejecimiento y los trastornos estéticos: una revisión
}

integradora

Recebido: 07/09/2021 | Revisado: 13/09/2021 | Aceito: 17/09/2021 | Publicado: 18/09/2021

\author{
Olicélia Magna Tunico De Oliveira \\ ORCID: https://orcid.org/0000-0001-9652-5116 \\ Faculdades Nova Esperança de Mossoró, Brasil \\ E-mail: olicelia.olvr@gmail.com \\ José Lucas Saraiva Nunes \\ ORCID: https://orcid.org/0000-0003-4892-6913 \\ Faculdades Nova Esperança de Mossoró, Brasil \\ E-mail: lucas.saraiva2013@gmail.com \\ Raissa Stephem Félix De Oliveira \\ ORCID: https://orcid.org/0000-0001-5571-7699 \\ Faculdades Nova Esperança de Mossoró, Brasil \\ E-mail: raissa_stephem@hotmail.com \\ Maria Emiliane Sousa De Oliveira \\ ORCID: https://orcid.org/0000-0001-8338-1694 \\ Faculdades Nova Esperança de Mossoró, Brasil \\ E-mail: emilianesouza2016@gmail.com \\ Lidiane Pinto De Mendonça \\ ORCID: https://orcid.org/0000-0001-5597-2446 \\ Faculdades Nova Esperança de Mossoró, Brasil \\ E-mail: lidianemendonca@facenemossoro.com.br
}

\begin{abstract}
Resumo
O crescimento pela boa forma e pela beleza vem, no entanto, extrair o foco da verdadeira importância para o nosso corpo de uma alimentação adequada, mostrando que hábitos inadequados, com alimentação inadequada, gera consequências para o metabolismo, levando a deficiência nutricional e obesidade. De uma maneira mais explicativa, garantir que a ingestão dos nutrientes aconteça na quantidade certa, assim a ausência de um deles pode causar um desequilíbrio metabólico, resultando em diversas desordens estéticas. Estudos comprovam que o envelhecimento e desordens estéticas estão relacionados com a qualidade de vida que se leva, pois, para se ter longevidade é preciso ter consciência de bons hábitos alimentares, Assim foi sugerida a seguinte pergunta como problematização para o projeto de pesquisa: Qual a influência da alimentação no envelhecimento e acometimento de desordens estéticas? Objetiva-se com essa pesquisa verificar a influência da alimentação no envelhecimento e nas desordens estéticas através de uma revisão integrativa. A metodologia aplicada foi uma pesquisa integrativa de literatura, de natureza qualitativa e exploratória, analisando artigos publicados em periódicos indexados que tratam sobre o assunto. Como resultados, foram a princípio encontrados 155 arquivos que acertaram pela seleção dos filtros de busca, segundo critérios préestabelecidos de inclusão. Da filtragem preliminar, resultaram 20 artigos elegíveis para avaliação dos títulos e resumos, entre estes foram excluídos 12 artigos que foram excluídos por apresentarem repetidos e resumos de livros e que não satisfaziam ao estudo em questão. Restando assim 8 artigos para análise e inclusão neste estudo.
\end{abstract}

Palavras-chave: Corpo; Beleza; Estética; Alimentação; Nutrientes; Qualidade de vida.

\begin{abstract}
Growth through good shape and beauty, however, extracts the focus of the true importance for our body of adequate nutrition, showing that inadequate habits, with inadequate nutrition, have consequences for the metabolism, leading to nutritional deficiency and obesity. In a more explanatory way, ensure that the intake of nutrients happens in the right amount, so the absence of one of them can cause a metabolic imbalance, resulting in various aesthetic disorders. Studies show that aging and esthetic disorders are related to the quality of life that one leads, because, in order to have longevity, it is necessary to be aware of good eating habits. Thus, the following question was suggested as a problematization for the research project: What is influence of food on aging and the onset of aesthetic disorders? The aim of this research is to verify the influence of food on aging and aesthetic disorders through an integrative review. The methodology applied was an integrative literature research, qualitative and exploratory in nature, analyzing articles published in indexed journals dealing with the subject. As a result, 155 files were initially found that were right by selecting the search filters, according to pre-established inclusion criteria. From the preliminary filtering, 20 articles were eligible for evaluation of titles and abstracts, among these, 12 articles were excluded that were excluded for presenting repeated
\end{abstract}


and book summaries and that did not satisfy the study in question. This leaves 8 articles for analysis and inclusion in this study.

Keywords: Body; Beauty; Aesthetics; Food; Nutrients; Quality of life.

\section{Resumen}

El crecimiento a través de la buena forma y la belleza, sin embargo, extrae el foco de la verdadera importancia para nuestro cuerpo de una nutrición adecuada, mostrando que los hábitos inadecuados, con una nutrición inadecuada, tienen consecuencias para el metabolismo, conduciendo a la deficiencia nutricional y la obesidad. De forma más explicativa, procurar que la ingesta de nutrientes se produzca en la cantidad adecuada, por lo que la ausencia de uno de ellos puede provocar un desequilibrio metabólico, dando lugar a diversos trastornos estéticos. Los estudios muestran que el envejecimiento y los trastornos estéticos se relacionan con la calidad de vida que se lleva, pues para tener longevidad es necesario estar atento a los buenos hábitos alimenticios, por lo que se sugirió la siguiente pregunta como una problematización para la investigación. proyecto: ¿Cuál es la influencia de la alimentación en el envejecimiento y la aparición de trastornos estéticos? El objetivo de esta investigación es verificar la influencia de los alimentos en el envejecimiento y los trastornos estéticos a través de una revisión integradora. La metodología aplicada fue una investigación bibliográfica integradora, de carácter cualitativo y exploratorio, analizando artículos publicados en revistas indexadas que tratan el tema. Como resultado, inicialmente se encontraron 155 archivos que eran correctos al seleccionar los filtros de búsqueda, de acuerdo con los criterios de inclusión preestablecidos. Del filtrado preliminar, 20 artículos fueron elegibles para evaluación de títulos y resúmenes, entre estos, se excluyeron 12 artículos que fueron excluidos por presentar resúmenes repetidos y de libros y que no cumplieron con el estudio en cuestión. Esto deja 8 artículos para su análisis e inclusión en este estudio.

Palabras clave: Cuerpo; Belleza; Estética; Alimentación; Nutrientes; Calidad de vida.

\section{Introdução}

O envelhecimento intrínseco é progressivo, previsível e inevitável, sendo determinado pela genética e derivado da diminuição da elastina, o que leva à atrofia, ao aparecimento de rugas e ao ressecamento. Já o envelhecimento extrínseco é um conjunto de mudanças advindas de fatores ambientais, e a pele se apresenta com rugas profundas, flacidez e sulcos (Pujol, 2011).

Os radicais livres podem ser gerados por meio de fontes endógenos, como inflamação e respiração aeróbica, ou por meio de fontes exógenos, como radiação ultravioleta, medicamentos e tabagismo (Pereira; Vidal; Constant, 2009). Os antioxidantes obtidos pela alimentação desaceleram o processo de envelhecimento, combatendo ou inativando esses radicais livres, que aparecem mesmo com as defesas naturais do corpo, provocando reações antes de ser eliminados (Ribeiro, 2010).

A investigação desenfreada pela boa forma e pela beleza vem, no entanto, extrair o foco da verdadeira importância para o nosso corpo de uma alimentação equilibrada. Nessa direção, diversos estudos já mostraram que maus hábitos alimentares, com deficiências nutricionais e excesso de gordura saturada, carboidratos simples e sódio, podem causar diversas alterações estéticas (Pereira; Vidal; Constant, 2009).

A falta de alguns nutrientes pode levar a um desequilíbrio metabólico, por isso, é importante o consumo em quantidades adequadas, pois resulta em diversas desordens estéticas que podem acometer: unhas, cabelos, pele, acne, manchas de pele, celulite, flacidez, estrias, gordura localizada e envelhecimento precoce. Também pode desenvolver problemas como baixo peso e até mesmo sobre peso e obesidade. (Jay, 1998, p. 71-77).

Nas últimas décadas, foi possível observar mudanças nos hábitos alimentares em diversos países, o que reflete a complexidade dos modelos de consumo e dos fatores que os determinam. Tais mudanças afetam a qualidade dos alimentos produzidos e industrializados. Na tentativa de adequar a alimentação ao ritmo acelerado do dia-a-dia, as escolhas e os hábitos de consumo passaram a apontar para alimentos mais condizentes com o novo estilo de vida, fazendo com que fossem incorporados hábitos rápidos e práticos (Pinheiro, 2001).

Neste contexto, os alimentos priorizados são aqueles ricos em antioxidantes, que podem variar desde frutas, verduras, fibras e alguns outros alimentos que sejam ricos em antioxidantes, como por exemplo, frutas cítricas, frutas vermelhas, aveia, linhaça e suco de uva integral. 
Diante desta preocupação com o corpo, a imagem corporal se torna um tema de interesse da nossa sociedade, sendo tão preponderante que leva as pessoas a se preocuparem excessivamente com ela. Desse ponto de vista, a noção de estética assola, já que, sobretudo na sociedade atual, ela é determinada por padrões culturais que variará de acordo com cada concepção de mundo, fazendo com que haja um corpo típico para cada sociedade. É esse corpo que pode variar de acordo com o contexto histórico e cultural, é o corpo que entra e sai da moda. (Alcântara 2001, p. 231-234).

Dessa forma, a nutrição estética, atua na melhoria da saúde como um todo, como também foca na melhora de desordens estéticas, dependendo do caso isso pode acontecer através de uma alimentação especial e de suplementação específica (Goldenberg, 2005).

Levando em consideração o processo de envelhecimento na atualidade, é necessários estudos que forneçam informações de forma sintetizada acerca da alimentação e disfunções estética e o grau de envelhecimento, estudos estes, pelos conhecimentos obtidos diante dos dados e arquivos relacionados ao tema trabalhado e fundamentado diante do envelhecimento precoce e desordens estéticas na atualidade (Goldenberg, 2005).

Destarte, o objetivo geral é verificar a influência da alimentação no envelhecimento e desordens estéticas através de uma revisão integrativa.

\section{Metodologia}

A metodologia aplicada para fundamentação desta pesquisa foi uma pesquisa integrativa de literatura, de natureza qualitativa e exploratória, analisando artigos publicados em periódicos indexados que tratam sobre o assunto, em bases de dados específicas, que abordam a temática.

Para Silva e Menezes (2000, p. 20), “a pesquisa qualitativa considera que há uma relação dinâmica entre o mundo real e o sujeito, isto é, um vínculo indissociável entre o mundo objetivo e a subjetividade do sujeito que não pode ser traduzido em números.

O presente trabalho tem natureza qualitativa com viés quantitativo. O estudo caracteriza-se como uma revisão narrativa da literatura para relacionar o padrão alimentar e estilo de vida ao processo de envelhecimento e das desordens estéticas (Pereira et. al., 2018).

Para a seleção dos estudos foi realizada uma busca de publicações indexadas nas bases de dados SciElo, LILACS e Pubmed, utilizando-se a combinação dos termos cadastrados no site DeCS (Descritores em Ciências da Saúde) “Consumo alimentar, estilo de vida, envelhecimento, estética, desordens".

A presente pesquisa não necessita de submissão ao comitê de ética em pesquisas em seres humanos, levando em consideração que na sua metodologia usam-se dados de domínio público.

O estudo em questão busca achados que comprovem através de estudos científicos a influência da alimentação no envelhecimento e desordens estéticas. Além disso, pode proporcionar a população uma síntese de estudos que mostrem a importância de uma alimentação saudável no processo de envelhecimento e na estética. A presente pesquisa não apresenta riscos.

Os resultados da pesquisa narrativa servirão como instrumento de comprovação de conclusão do curso de nutrição da Faculdade Nova Esperança de Mossoró. Há a pretensão de publicação em revistas para que possam estar exposto ao público os achados da pesquisa que poderão ser relevantes para estudos posteriores. Todos os custos da pesquisa foram de responsabilidade dos pesquisadores envolvidos no estudo.

Para responder a pergunta deste artigo foi utilizada a estratégia PICO de pesquisa conforme o Quadro 1 abaixo. 
Quadro 1: PICO.

\begin{tabular}{|c|l|}
\hline ACRÔNIMO & \multicolumn{1}{|c|}{ DESCRIÇÃO } \\
\hline $\mathrm{P}$ & Pessoas vulneráveis a mau consumo alimentar. \\
\hline $\mathrm{I}$ & Reunir estudos e pesquisas acerca do estilo de vida das pessoas. \\
\hline $\mathrm{C}$ & $\begin{array}{l}\text { Comparar pessoas vulneráveis, suas patologias e associar ao } \\
\text { envelhecimento por meio da má alimentação. }\end{array}$ \\
\hline $\mathrm{O}$ & Pessoas vulneráveis a estética e as desordens alimentares. \\
\hline
\end{tabular}

Fonte: Desenvolvido pelos autores (2021).

A estratégia PICO (acrônimo para P: população/pacientes; I: intervenção; C: comparação/controle; O: desfecho/Resultado) é utilizada para auxiliar o que de fato a pergunta de pesquisa deve especificar.

No início da busca foram encontrados 20 trabalhos nas bases de dados SCIELO; LILACS e PUBMED sobre o assunto, após as diversas análises sobre A Influência da Alimentação no Envelhecimento e nas Desordens Estéticas.

Os critérios de inclusão são estudos científicos que discorram sobre o tema, selecionados nas línguas portuguesa e inglesa, publicados nos últimos 10 anos. Os critérios de exclusão foram: Artigos que não apresentassem os critérios de seleção mencionados anteriormente, assim como, teses, dissertações ou artigos que não atendessem aos objetivos propostos. Foram incluídos efetivamente para revisão 8 estudos como mostrado no fluxograma PRISMA no Quadro 2.

Quadro 2. Fluxograma Prisma e a descrições das buscas nas bases de dados:

\begin{tabular}{|c|c|}
\hline \multicolumn{2}{|c|}{$\begin{array}{c}\text { Busca pelos descritores nas bases } \\
\text { SCIELO, LILACS E PUBMED: } \\
155 \text { Artigos }\end{array}$} \\
\hline Após leitura de título e resumo: & $\begin{array}{c}\text { Artigos que foram excluídos Por } \\
\text { Apresentarem repetidos e resumos de } \\
\text { livros e que não satisfaziam ao estudo em } \\
\text { questão: }\end{array}$ \\
& $\begin{array}{c}\text { 12 Artigos } \\
\text { Compuseram a Revisão Integrativa: } \\
\text { 8 artigos }\end{array}$ \\
\hline
\end{tabular}

Fonte: Desenvolvido pelos autores (2021).

Por fim, os totais de artigos recuperados pela tática de busca foram basicamente filtrados pela leitura de seus títulos.

\section{Resultados}

Foram a princípio encontrados 155 arquivos que acertaram pela seleção dos filtros de busca, segundo critérios préestabelecidos de inclusão. Da filtragem preliminar, resultaram 20 artigos elegíveis para avaliação dos títulos e resumos, entre estes foram excluídos 12 artigos que foram excluídos por apresentarem repetidos e resumos de livros e que não satisfaziam ao 
estudo em questão. Restando assim 8 artigos para análise e inclusão neste estudo, conforme processo de seleção apresentado no Quadro 3.

Quadro 3. Pesquisa bibliográfica e seleção dos artigos.

\begin{tabular}{|c|c|c|c|c|c|c|}
\hline & \multicolumn{2}{|c|}{ SCIELO } & \multicolumn{2}{c|}{ LILACS } & \multicolumn{2}{c|}{ PUBMED } \\
\hline Descritores & Encontrados & Utilizados & Encontrados & Utilizados & Encontrados & Utilizados \\
\hline $\begin{array}{c}\text { Consumo } \\
\text { Alimentar }\end{array}$ & 20 & 2 & 20 & 1 & 10 & 0 \\
\hline Estilo de Vida & 20 & 1 & 30 & 1 & 10 & 0 \\
\hline Envelhecimento & 5 & 1 & 5 & 0 & 5 & 0 \\
\hline Estética & 5 & 0 & 5 & 0 & 5 & 1 \\
\hline Desordens & 5 & 0 & 5 & 0 & 5 & 1 \\
\hline TOTAL & 55 & 4 & 65 & 2 & 35 & 2 \\
\hline
\end{tabular}

Fonte: Desenvolvido pelos autores (2021).

A amostra final para o estudo foi constituída dos 8 artigos sumarizados no Quadro 4.

Quadro 4. Artigos selecionados.

\begin{tabular}{|c|c|c|c|c|}
\hline & ARTIGO & OBJETIVO & $\begin{array}{c}\text { METODOL } \\
\text { OGIA }\end{array}$ & CONCLUSÃO \\
\hline 1 & $\begin{array}{l}\text { (MONTEIRO; MONTEIRO, 2013; } \\
\text { FONTELES, SANTOS; SILVA, } \\
\text { 2009). } \\
\text { Fisiologia do envelhecimento: } \\
\text { processos e alterações no organismo. }\end{array}$ & $\begin{array}{c}\text { Mostrar as variações e } \\
\text { mudanças biológicas, } \\
\text { fisiológicas e anatômicas que } \\
\text { o ser humano sofre ao longo } \\
\text { dos anos. }\end{array}$ & Relatório & $\begin{array}{l}\text { Descrever a } r \\
\text { pela longevidade, e um } \\
\text { envelhecimento com qualidade de } \\
\text { vida, apresentando um fator } \\
\text { desafiador nos dias de hoje. }\end{array}$ \\
\hline 2 & $\begin{array}{l}\text { (ORIÁ } \text { et. al., 2003). } \\
\text { Estudo das alterações relacionadas } \\
\text { com a idade na pele humana, } \\
\text { utilizando métodos de histo- } \\
\text { morfometria e autofluorescência. }\end{array}$ & $\begin{array}{l}\text { Analisar as alterações da pele } \\
\text { normal com o envelhecimento } \\
\text { mediante estratégias de } \\
\text { histomorfometria e } \\
\text { autofluorescência. }\end{array}$ & $\begin{array}{c}\text { Revisão } \\
\text { Integrativa }\end{array}$ & $\begin{array}{l}\text { Como reflexão, o envelhecimento } \\
\text { propaga à redução da espessura } \\
\text { da derme e epiderme, bem como } \\
\text { ao aplanamento da junção dermo- } \\
\text { epidérmica. As mudanças da pele } \\
\text { ao longo da vida nem sempre } \\
\text { seguem um perfil linear, } \\
\text { mostrando drásticas alterações } \\
\text { nas últimas décadas de vida. }\end{array}$ \\
\hline 3 & $\begin{array}{c}\text { (OLIVEIRA, 2008). } \\
\text { Considerações atuais sobre a } \\
\text { vitamina D }\end{array}$ & $\begin{array}{l}\text { Discutir a fisiologia da } \\
\text { vitamina } \mathrm{D} \text { em prol de um } \\
\text { bom estilo de vida. }\end{array}$ & $\begin{array}{c}\text { Revisão } \\
\text { Bibliográfica }\end{array}$ & $\begin{array}{l}\text { Descrever como a vitamina D é } \\
\text { um hormônio extremamente } \\
\text { importante, tanto para o } \\
\text { metabolismo ósseo, como para } \\
\text { outras funções, que são cada vez } \\
\text { mais estudadas. }\end{array}$ \\
\hline 4 & (DIAS et. al., 2017). & $\begin{array}{c}\text { Discutir a relação entre } \\
\text { adesão à prática da atividade } \\
\text { física, desordens estéticas e a } \\
\text { funcionalidade do idoso em } \\
\text { prol de um bom estilo de } \\
\text { vida. }\end{array}$ & $\begin{array}{c}\text { Revisão } \\
\text { Bibliográfica }\end{array}$ & $\begin{array}{l}\text { A reflexão diz que população } \\
\text { idosa tem aumentado } \\
\text { consideravelmente em todo o } \\
\text { mundo. Para tanto, se faz } \\
\text { necessário compreender mais } \\
\text { detalhadamente os fatores } \\
\text { associados às desordens estéticas. }\end{array}$ \\
\hline
\end{tabular}




\begin{tabular}{|c|c|c|c|c|}
\hline 5 & $\begin{array}{l}\text { (HARRIS, 2009). } \\
\text { Mudanças no padrão de consumo } \\
\text { alimentar no Brasil e no mundo. }\end{array}$ & $\begin{array}{l}\text { Compreender o consumo de } \\
\text { alimentos no Brasil e } \\
\text { comprovar que ele é } \\
\text { cointegrado com o do resto do } \\
\text { mundo. }\end{array}$ & $\begin{array}{c}\text { Revisão } \\
\text { Bibliográfica }\end{array}$ & $\begin{array}{c}\text { Na descrição foi possível } \\
\text { constatar que, pelo menos no } \\
\text { Brasil, o consumo alimentar não } \\
\text { são cointegrados, não existindo } \\
\text { uma relação de equilíbrio no } \\
\text { longo prazo. Provocando assim } \\
\text { até o envelhecimento precoce. }\end{array}$ \\
\hline 6 & $\begin{array}{c}\text { (VINHOLES } \text { et. al., 2009, p.791- } \\
\text { 799). } \\
\text { Alimentos Anti-inflamatórios } \\
\text { auxiliam a Estética Corporal. }\end{array}$ & $\begin{array}{l}\text { Compreender os alimentos } \\
\text { que pode beneficiar na } \\
\text { estética corporal. }\end{array}$ & $\begin{array}{c}\text { Revisão } \\
\text { Bibliográfica }\end{array}$ & $\begin{array}{l}\text { Descrever sobre os hábitos que } \\
\text { podem beneficiar a estética } \\
\text { corporal é extremamente } \\
\text { importante para a questão do } \\
\text { envelhecimento. }\end{array}$ \\
\hline 7 & $\begin{array}{l}\text { (STAVRIC, 1994; FOTSIS ET AL., } \\
\text { 1997; POOL-ZOBEL ET AL., 1997). } \\
\text { Radicais livres e os principais } \\
\text { antioxidantes da dieta. }\end{array}$ & $\begin{array}{l}\text { Analisar a importância dos } \\
\text { antioxidantes da dieta sobre as } \\
\text { estratégias de defesa dos } \\
\text { organismos contra os radicais } \\
\text { livres. }\end{array}$ & $\begin{array}{c}\text { Revisão } \\
\text { Bibliográfica }\end{array}$ & $\begin{array}{l}\text { A carência de benefícios, e até } \\
\text { mesmo prejuízo na suplementação } \\
\text { com vitaminas sobre o } \\
\text { desenvolvimento de diferentes } \\
\text { tipos de doenças até crônicas. } \\
\text { Sendo imprescindíveis mais } \\
\text { estudos sobre o mecanismo de } \\
\text { ação desses agentes antes da sua } \\
\text { prescrição em larga escala. }\end{array}$ \\
\hline 8 & $\begin{array}{l}\text { (JACOB, 1995; NIKI ET AL., 1995; } \\
\text { HERCBERG et. al., 1998). } \\
\text { Análise dos fatores de risco para o } \\
\text { envelhecimento da pele: aspectos } \\
\text { nutricionais. }\end{array}$ & $\begin{array}{l}\text { Descrever a relação da } \\
\text { ingestão dietética com o } \\
\text { envelhecimento cutâneo. }\end{array}$ & $\begin{array}{c}\text { Revisão } \\
\text { Bibliográfica }\end{array}$ & $\begin{array}{l}\text { A nutrição compensada é um } \\
\text { ambiente chave para a } \\
\text { conservação da saúde e da beleza } \\
\text { da pele e o excepcional fator } \\
\text { versus o envelhecimento que } \\
\text { pode ser modificado. }\end{array}$ \\
\hline
\end{tabular}

Fonte: Desenvolvido pelos autores (2021).

\section{Discussão}

O envelhecimento ocorre de forma natural ou biológico, no qual ocorrem várias alterações fisiológicas e funcionais, mudanças no metabolismo celular e no estado nutricional, acarretando não só alteração na composição corporal, mas tornandoo mais vulnerável ao desenvolvimento de doenças crônicas (Monteiro; Monteiro, 2013; Fonteles, Santos; Silva, 2009).

A mudança nas características da pele durante o envelhecimento é frequentemente determinada por fontes ambientais assim como por fatores intrínsecos, alguns deles está relacionado com a desestruturação do tecido conjuntivo da derme. Alguns sinais mais visíveis ocasionados por alterações de uma pele é a própria velhice levando a atrofia, enrugamento. Alterações no tecido conjuntivo, o qual atua é um grande alicerce estrutural para a epiderme, delineiam mudanças na aparência externa, que são refletidas no estrato córneo. As modificações do colágeno - elástico ao longo da vida estabelecem uma base morfológica substancial para compreender as adaptações bioquímicas e biomecânicas da pele com a idade. A espessura da pele e suas propriedades viscoelásticas não dependem apenas da quantidade de material presente na derme, mas também, de sua organização estrutural (Oriá et. al., 2003).

A síntese de vitamina $\mathrm{D}$, que é a principal função endócrina na pele é comprometida com a idade devido à exposição insuficiente ao sol ou por deficiência desta vitamina na ingestão de alguns alimentos. Levando a comprometer algumas mudanças na derme, como a perda de aproximadamente $20 \%$ da espessura da pele, devido à redução na síntese e secreção das fibras colágenas e elasticidade, causada pela diminuição da atividade metabólica dos fibroblastos. Há também diminuição das células de sustentação, o colágeno é formado tornando as fibras mais espessas e as fibras elásticas perdem parte de sua estrutura, tornando flácida (Oliveira, 2008). 
Portanto, alguns comportamentos adotados pelos indivíduos devem valorizar princípios que contribuam á hábitos de vida mais saudável que promova o desenvolvimento de doenças. Um desses princípios parte muito de suas escolhas por alguns alimentos, das atividades do dia a dia, dos relacionamentos sociais, do controle de atividade física diário, stress (Dias et. al., 2017).

Gomes e Bezerra (2009), afirma que os "nutracêuticos, ricos em determinados grupos químicos, possuem ação comprovada no combate a alguns tipos de câncer", comprovando mais uma vez a sua capacidade de promover a saúde e a longevidade.

Diante deste contexto, o atual consumo alimentar de diversos países, dentre eles o Brasil, vem sofrendo rápidas modificações nas últimas décadas, influenciadas pelas mudanças socioeconômicas e demográficas. Em paralelo ao aumento da ingestão de alimentos ultra processados e ricos em açúcar e aditivos químicos, a prevalência de obesidade e doenças crônicas, incluindo aquelas associadas à estética, também, têm aumentado de forma alarmante em toda a população. Dessa maneira, ao longo dos anos, a alimentação no mundo ocidental tem se modificado drasticamente. É fato que grande parte das desordens é de caráter nutricional, e há aquelas que acabam afetando a estética do indivíduo (HarriS, 2009).

A beleza vem sendo destaque global para o cuidado estético, que vem crescendo cada vez mais. Tornando um conjunto no qual a nutrição tem um papel principal na prevenção e tratamento para obtenção em saúde e ao mesmo tempo da estética.

Quando se fala em tratamento estético, deve-se partir do princípio de uma alimentação antiinflamatória, uma vez que a alimentação habitual (carnes gordas, leite integral, alimentos refinados, doces, bebidas alcoólicas, embutidos e enlatados), são pró-inflamatórios, ou seja, favorecem o aparecimento da inflamação. Vale lembrar que a inflamação contínua (crônica) pode causar alterações celulares, e contribuir para o surgimento de algumas doenças. (Vinholes et. al., 2009, p.791-799).

Estudo realizado por Liberato e colaboradores (2006), verificou que práticas alimentares inadequadas com alto consumo energético pode ocorrer falhas no metabolismo, levando à obesidade e deficiências nutricionais, e isso tem afetado mais de 2 bilhões de pessoas a nível mundial. Isso ocorre decorrente ao alto consumo de alimentos industrializados que está em alto consumo cada vez mais.

As estrias são lesões tegumentares adquiridas e, como toda cicatriz, possuem difícil tratamento. Com o avanço atual das técnicas, vários tratamentos estéticos são realizados, porém, não há estudos que comprovem sua eficácia para o desaparecimento total das estrias e, por tratar-se de predisposição genética, a prevenção inclui tanto a manutenção do peso ideal quanto uma boa hidratação cutânea.

Estudo analisado por Amaral (2018), trazem como resultados que os tratamentos estéticos realizados para estrias são classificados de acordo com a lesão e o grau apresentado. As estrias quando tratadas no início do seu aparecimento apresentamse com coloração avermelhada, onde autores afirmam ser a fase mais efetiva para obtenção de uma resposta terapêutica, seja com a utilização de peelings e/ou com a galvanopuntura, pelo aumento da vascularização local. Simultaneamente a estes tratamentos, o paciente pode utilizar os cosmecêuticos (Hydroxyprolisilane $\mathrm{CN}$ ), que também estimulará a produção de matriz extracelular, além de hidratar toda a região para evitar o surgimento de novas estrias, uma vez que é uma excelente opção para uso domiciliar.

Os antioxidantes obtidos da dieta, como as vitaminas C, E e A, os flavonóides e carotenóides são extremamente importantes para impedir a presença dos radicais livres em nosso corpo. Os alimentos contêm compostos oxidantes, os quais podem ocorrer naturalmente ou ser introduzidos durante o processamento para o consumo (Waters et al., 1996). O estudo relata que por outro lado, os alimentos, principalmente as frutas, verduras e legumes, são agentes antioxidantes, tais como as vitaminas C, E e A, a clorofilina, os flavonóides, carotenóides, curcumina e outros que são capazes de restringir a propagação das reações em cadeia e as lesões induzidas pelos radicais livres (Stavric, 1994; Fotsis Et Al., 1997; Pool-Zobel et al., 1997). 
A utilização de compostos antioxidantes encontrados na dieta ou mesmo sintéticos é um dos mecanismos de defesa contra os radicais livres que podem ser empregados em indústrias de alimentos, cosméticos, bebidas e também na medicina, sendo que muitas vezes os próprios medicamentos aumentam a geração intracelular desses radicais livres (Doroshow, 1983; Halliwell et al., 1995; Weijl et. al., 1997).

O antioxidante possui ativos principais no atual uso como nutrientes isolados no tratamento e prevenção de doenças. Entretanto, em alimentos são encontrados uma ampla variedade dessas substâncias que auxiliam como proteção das células e dos tecidos e entrada de elementos estranhos (Jacob, 1995; Niki et al., 1995; Hercberg et. al., 1998).

A alimentação tem ponto importante na dieta como influenciadora de forma direta na gravidade das rugas faciais, portanto, uma dieta saudável está associada a menos enrugamento, onde uma dieta pouco saudável aumenta esse procedimento. Com isso, cria melhoras e hábitos na qualidade de vida nas mulheres para se cuidar, através da alimentação equilibrada e manterse com aparência mais jovem. (Obbagy et al., 2019).

Uma Pesquisa e Desenvolvimento, Colworth Science Park, Bedfordshirec; e Leiden University College, propôs testes com alimentos que possa promover o não aparecimento de rugas faciais em mulheres. Neste estudo foram associados que alimentos como vegetais ricos em caroteno, vitamina $\mathrm{C}$, azeite e peixes estão associados a menos foto envelhecimento na pele, então, o estudo relata que o consumo de gorduras saturadas e açúcar estão associados a mais enrugamento facial.

Vários fatores que ocasiona os novos fatores de risco conhecidos e possíveis (física atividade, ingestão diária de energia) nas rugas faciais. Outras variais de estudo foram analisados com Holandeses, faz análise de holandês Índice de dieta saudável (DHDI) e outra análise componente principal análise (PCA) para investigar o parâmetro maior de rugas e estilo de vida saudável na grande população, foram testados para avaliar rugas e dieta. Recomendações nutricionais, na dieta saudável é aplicado para melhorar tanto o aspecto jovem da pele como também, a saúde no geral.

Indivíduos que se alimentam com soja e vegetais de cor amarela, possuem menos chances de desenvolver rugas faciais. Portanto esse grupo de alimentos isolados de seus nutrientes em si mostra resultados positivos como negativos. Pois o é importante avaliar todos esses parâmetros de nutrição em grupos de alimentos associados.

O mecanismo biológico responsável pela associação entre rugas da pele e dieta não saudável poderia ser o aumento da carga de estresse oxidativo, um estado inflamatório regulado positivamente, ou o efeito de produtos finais de glicação avançada, que podem interromper no metabolismo celular e enfraquece a rede protetora dos antioxidantes.

A celulite é uma condição fisiológica, característica das mulheres, com muitos fatores envolvidos, mas desconhecidos. Deste modo, a melhor forma de se prevenir é investir em hábitos saudáveis, tais como: ingerir uma grande quantidade de água diariamente para melhorar a circulação sanguínea; diminuir a ingestão de sódio, que pode causar retenção hídrica; evitar alimentos gordurosos e muito doces, como os das redes de fast-food; parar de fumar; prática regular de atividade física, além de reduzir a gordura corporal e diminuir as medidas antropométricas, o que pode aumentar o problema. (Monteiro et al., 2019).

É preciso entender que nenhum tratamento estético para celulite e flacidez funciona sem alterações nos hábitos alimentares e no estilo de vida, eles estão associados. É preciso reduzir o consumo de açúcar e gorduras, ingerir muita água e a prática de exercícios físicos, como os aeróbicos, que ajudam na redução da gordura corporal, ou a musculação, que auxilia na atrofia da pele e musculo. Concluímos que o tratamento da celulite é muito difícil e precisa ser aliado com combinação de tratamentos nutricional e estéticos e grande adesão do paciente à mudança de hábitos e de estilo de vida para adesão de um tratamento mais aperfeiçoado.

A celulite atinge cerca de $95 \%$ das mulheres, embora é mais atingida em mulheres com pele brancas. Sendo pouco desenvolvida em homens, mas podendo ocorrer quando houver algum desequilíbrio de hormônios. Podendo ocorrer entre as regiões como nos quadris, coxas e nádegas; mamas, abdome, braços. Não há uma causa especifica que seja comprovada, sendo que o fator genético é importante. Problemas circulatórios, alterações hormonais, pílula anticoncepcional também pode 
desencadear esse problema. Hábitos de vida inadequados, uma alimentação rica em açúcar simples, o sedentarismo, a tensão emocional e o excesso de toxinas no organismo contribuem para o aparecimento da celulite.

Uma dieta balanceada e bem orientada pode melhorar o aspecto da celulite pela redução da gordura. Muitos suplementos alimentares e misturas herbais estão disponíveis no mercado, com diversos apelos de benefícios, mas sem nenhuma comprovação até o momento.

\section{Considerações Finais}

Nota-se a importância da nutrição no processo das funcionalidades na ciência de uma busca por vida saudável que traz uma beleza duradoura, e ao mesmo tempo, que se leva em consideração o envelhecimento cutâneo. O padrão alimentar tem influência positiva como negativa nas desordens estéticas que acometem uma população. Mostrando grande realidade, pois, através dos hábitos de vida, podem transformar o corpo e o metabolismo celular, levando a várias desestruturações em enzimas e no DNA.

Portanto, percebe-se da importância dos resultados encontrados no decorrer deste trabalho, que servirá de fonte de pesquisa para futuros pesquisadores e estudantes, ao buscarem base para suas futuras produções acadêmicas.

\section{Referências}

Amaral, C. N., et. al. (2018) Tratamento em estrias: Um levantamento teórico da microdermoabrasão e do peeling químico.12 f. Trabalho de Conclusão de Curso (Cosmetologia e Estética) -Universidade do Vale do Itajaí, Balneário Camboriú.

Alcântra, M. L. B. (2001). O corpo do brasileiro: estudos de estética e beleza. Rev Antropol; 44 (2): 231-234. Carvalho, G.F. et al. Avaliação dos efeitos da radio frequência no tecido conjuntivo. http://www.moreirajr.com.br/revistas.asp?fase=r003\&id_materia=4555.

Cerutti, P. A. (1994). Oxidant stress and carcinogenesis. European Journal of Clinical Investigation, Oxford, 21(1), 1-5, 1991. Cerutti, P.A. Oxy-radicals and cancer. Lancet, London, 344(8926), 862-863.

Dias, A. C. S., et. al. (2017). Efeito microscópico do dióxido de carbono na atrofialinear cutânea. Monografia apresentada ao Curso de Fisioterapia do Centro de Ciências Biológicas e da Saúde da UNAMA. Belém.

Doroshow, J. H. (1983). Effect of anthracycline antibiotics on oxygen radical formation in rat heart. Cancer Research, Baltimore, 43(2), 460-472.

Faé, C, Liberali, R, \& Coutinho, V. F. (2015). Deficiência de nutrientes a longo prazo no pós-operatório de cirurgia bariátrica - revisão sistemática. Sa Bios: Rev. Saúde e Biol.10(2):46-53.

Fotsis, T., et al. (1997). Flavonoids, dietary-derived inhibitors of cell proliferation and in vitro angiogenesis. Cancer Research, Baltimore, 57(14), 2916-2921.

Goldenberg, M. Gênero e corpo brasileiro. Psicol Clín 2005; 17(2): 65-80.

Gil, A. C. (2002). Como elaborar projetos de pesquisa-(4a. ed.): Atlas. p.42

Harris, M. I. N. de C. (2009). Pele: estrutura, propriedades e envelhecimento. (3 ${ }^{\text {a }}$ ed.) revisada e ampliada. São Paulo: Editora Senac.

Jay, V., Berthon, J. Y., Hagege, P., Pouget, M. P., Lejeune, B., \& Pourrat, H. (1998). Cosm. Toiletries 113: 71-77.

Jacob, R. A. (1995). The integrated antioxidant system. Nutrition Research, New York, 15(5), 755-766.

Monteiro, P. K. S., Rosas, R. F. (2013). Efeitos da corrente galvânica no tratamento das estrias atróficas. Rio de Janeiro: Revinter.

Monteiro, C. A., Cannon, G., Lawrence, M., Louzada, M. L. da C., \& Machado, P. P. (2019). Ultra-processed foods, diet quality, and health using the NOVA classification system. FAO. http://www.wipo.int/amc/en/mediation/rules

NikI, E., Nogushi, N., Tsuchihashi, H., \& Gotoh, N. (1995). Interaction among vitamin C, vitamin E, and b-carotene. American Journal of Clinical Nutrition, Bethesda, 62(6), 1322-1326.

Obbagy, J. E., English, L. K., Psota, T. L., Wong, Y. P., Butte, N. F., Dewey, K. G., Fox, M. K., Greer, F. R., Krebs, N. F., Scanlon, K. S., \& Stoody, E. E. (2019). Complementary feeding and micronutrient status: A systematic review. In American Journal of Clinical Nutrition (Vol. 109, pp. 852S-871S). https://doi.org/10.1093/ajen/nqy266

Oliveira, A. L. (2008). Curso de estética. v. 2. São Paulo: Yendis. 
Research, Society and Development, v. 10, n. 12, e223101220333, 2021

(CC BY 4.0) | ISSN 2525-3409 | DOI: http://dx.doi.org/10.33448/rsd-v10i12.20333

Organização Pan-Americana de Saúde (OPAS). (2001). XXXVI Reunión del Comitê Asesor de Investigaciones en Salud - Encuesta Multicêntrica - Salud, Bienestar y Envejecimiento (SABE) en América Latina y el Caribe: informe preliminar.

Oriá, R. B., et al. (2013). Estudo das alterações relacionadas com a idade na pele humana, utilizando métodos de histo-morfometria e autoflorescência. In: Congresso Brasileiros de Dermatologia. Fortaleza: UFC, p. 459-463.

Pool-Zobel, B. L., Bub, A., Müller, H., Wollowski, I., \& Rechkemmer, G. (1997). Consumption of vegetables reduces genetic damage in humans: first results of a human intervention trial with carotenoid-rich foods. Carcinogenesis, New York, 18(9), 1847-1850.

Pinheiro, K. (2001). História da Alimentação. Universitas Ciências da saúde. 3: 173-190.

Pujol, A. P. (2011). Nutrição aplicada à estética: Editora Rúbio.

Pereira, A. L. F., et al. (2009). Antioxidantes alimentares: importância química e biológica. Nutrire: Rev. Soc. Bras. Alim. Nut., São Paulo, 34(3), 231-247, dez.

Pereira A. S., et al. (2018). Metodologia da pesquisa científica. [free e-book]. Santa Maria/RS. Ed. UAB/NTE/UFSM.

Pompella, A. (1997). Biochemistry and histochemistry of oxidant stress and lipid peroxidation. International Journal of Vitamin and Nutrition Research, Bern, 67(5), 289-297.

Ribeiro, C. J. (2006). Cosmetologia aplicada a Dermoestética. (1ª. ed.): Pharmabook.

Ribeiro, C. J. (2010). aplicada à dermoestética. (2 ${ }^{\mathrm{a}}$. ed.): Pharmabooks.

Silva, E. L. da., \& Menezes, E. M. (2001). Metodologia da pesquisa e elaboração de dissertação. Florianópolis: Laboratório de Ensino á Distância da UFSC.

Shephard. R. J. (2003). Envelhecimento, atividade física e saúde: Phorte.

Silva, E, A., et al. (2012). Terapia Capilar para o tratamento da alopecia androgenética masculina e alopecia areata. Florianopolis, Santa Catarina.

Stavric, B. (1994). Antimutagens and anticarcinogens in foods. Food Chemical Toxicology, Oxford, 32(1), 79-90.

Vinholes, D. B., Assunção, M. C. F., \& Neutzling, M. B. (2009). Frequência de hábitos saudáveis de alimentação medidos a partir dos 10 Passos da Alimentação Saudável do Ministério da Saúde. Pelotas, Rio Grande do Sul, Brasil, Cad. Saúde Pública. 25(4) 791-799.

Waters, M. D., et al. (1996). Activity profiles of antimutagens: in vitro and in vivo data. Mutation Research, Amsterdam, 350(1), 109-129. 